\title{
Dendropanax morbifera Extract Protects Cardiomyocytes against Hypoxia/ Reoxygenation Injury by Inhibition of Reactive Oxygen Species Generation and Calcium Perturbation
}

\author{
Leejin Lim ${ }^{1,2}$, Sujin $\mathrm{Ju}^{1}$, and Heesang Song ${ }^{1,3, *}$ \\ ${ }^{1}$ Depatment of Biomedical Sciences, Chosun University Graduate School, Gwangju 61452 Korea \\ ${ }^{2}$ Cancer Mutation Research Center, Chosun University, Gwangju 61452 Korea \\ ${ }^{3}$ Department of Biochemistry and Molecular Biology, Chosun University School of Medicine, Gwangju 61452 Korea
}

\begin{abstract}
Ischemia/reperfusion-induced myocardial injury is the main cause of acute myocardial infarction. Dendropanax morbifera Léveille has been used in traditional medicines for the treatment of various diseases such as headache, infectious diseases, and general debility. However, the effect of extract from D. morbifera (EDM) on myocardial ischemic injury is still unknown. In this study, the effects of EDM on neonatal rat cardiomyocytes with hypoxia/reoxygenation $(\mathrm{H} / \mathrm{R})$ injury were investigated. The viability of cardiomyocytes with $\mathrm{H}(30 \mathrm{~min}) / \mathrm{R}$ (1 h) decreased; however, treatment with EDM significantly inhibited H/R injury-induced cardiomyocyte death. Further, we observed that reactive oxygen species (ROS) generation and intracellular calcium concentration $\left(\mathrm{Ca}^{2+}{ }_{\mathrm{i}}\right)$ were significantly reduced in EDM-treated cardiomyocytes compared with that in H/R-injured positive control. In addition, western blotting results showed that EDM attenuated abnormal changes of RyR2 and SERCA2a genes in hypoxic cardiomyocytes. These results suggest that EDM ameliorates ROS generation and $\mathrm{Ca}^{2+}{ }_{i}$ homeostasis to prevent dysregulation of calcium regulatory proteins in the heart, thereby exerting cardioprotective effects and reducing hypoxia-induced cardiomyocyte damage, which verifies the potential use of EDM as a new therapeutic agent for the treatment of myocardial ischemic injury.
\end{abstract}

Keywords - Dendropanax morbifera, hypoxia/reoxygenation, cardiomyocytes

\section{Introduction}

Myocardial ischemia is caused by the occlusion of one or more coronary arteries, resulting in a decrease in oxygen supply to the myocardium. If the ischemic period is prolonged, cardiomyocyte death occurs, and the ischemic episode causes myocardial infarction (MI). ${ }^{1}$ Ischemia-reperfusion injury occurs when blood supply returns to an organ after a short period of ischemia, resulting in an "overgeneration" of reactive oxygen species (ROS) by mitochondria. ${ }^{2}$ The excessive production of ROS has been shown to contribute to pathological conditions, including cardiovascular disease (CVD), cancer, and even aging. ${ }^{3-5}$ In addition, ischemic conditions cause calcium $\left(\mathrm{Ca}^{2+}\right)$ overload in the myocardium leading to acceleration of heart damage. ${ }^{6}$

Many epidemiological studies have shown that diets

\footnotetext{
*Author for correspondence

Heesang Song, Department of Biochemistry and Molecular Biology,

Chosun University School of Medicine, 309 Pilmundaero, Dong-gu,

Gwangju 61452, Korea.

Tel: +82-62-230-6290; E-mail: hsong@chosun.ac.kr
}

rich in natural phenolic compounds reduce the risk of CVD. The plant Dendropanax morbifera Léveille belonging to the family Araliaceae is distributed throughout the southern part of Korea. ${ }^{7}$ It is used for the production of golden varnish. D. morbifera is also an effective folk medicine for the treatment of several conditions, such as infectious diseases, skin diseases, headaches, and other illnesses. ${ }^{7}$ Recently, polyacetylene compounds isolated from $D$. morbifera showed anticomplement activity. ${ }^{8}$ Subsequently, five compounds, oleifolioside A and B, dendropanoxide, $\alpha$ - and $\beta$-amyrin have been defined from D. morbifera and showed anti-diabetic, ${ }^{9}$ anti-atherogenic, ${ }^{10}$ anti-plasmodial, ${ }^{11}$ anti-complement, ${ }^{12}$ anti-inflammatory ${ }^{13}$ and anti-cancer ${ }^{14-16}$ effects. However, the effects of EDM on myocardial injury have not yet been reported. In the present study, we showed the attenuating effects of EDM on hypoxia/reoxygenation $(\mathrm{H} / \mathrm{R})$ injury caused by increased $\mathrm{ROS}$ and $\mathrm{Ca}^{2+}$ concentration in cardiomyocytes. 


\section{Experimental}

Preparation of extracts from D. morbifera-The plant material, which comprised the leaves or stem of $D$. morbifera, was collected in Jangheung province (batch no. 2014F) in Korea and then authenticated. Briefly, dried leaves $(1.4 \mathrm{~g})$ and stems $(21 \mathrm{~g})$ of D. morbifera were extracted with distilled water $(500 \mathrm{~mL})$ at $60^{\circ} \mathrm{C}$ for $24 \mathrm{~h}$. The primary extract was centrifuged at $700 \mathrm{~g}$ for $5 \mathrm{~min}$ to remove the plant material. The solution was filtered under sterile conditions of laminar flow. For the preparation of supercritical fluid extracts, an automated supercritical fluid extractor (ILSHIN AUTOCLAVE CO., LTD.) was used under extraction conditions ranging from 150 bar to 350 bar. In each extraction, $60 \mathrm{~g}$ of crushed dry D. morbifera leaves or stem (approximately $0.5 \mathrm{~mm}$ ) was used. In our extraction, $99.999 \% \mathrm{CO}_{2}$ was used to remove essence oil from powdered D. morbifera leaves. For preparation of fermented extracts, dried leaves and stems of D. morbifera were sterilized at $121^{\circ} \mathrm{C}$ for $30 \mathrm{~min}$. After cooling, the products were inoculated with Aspergillus oryzae (2\%) and incubated for $72 \mathrm{~h}$. All fermentations were carried out at $45^{\circ} \mathrm{C}$ and $70 \%$ relative humidity and fermented materials were further processed by water extraction method. The resultant EDM was stored at $4{ }^{\circ} \mathrm{C}$ and diluted by $50 \mathrm{X}$ in distilled water immediately before the experiments.

Primary culture of neonatal rat cardiomyocytes Neonatal rat cardiomyocytes were isolated and purified by the method by Vandergriff et al, with slight modifications. ${ }^{17}$ Briefly, 2- to 3-day-old Sprague-Dawley rat pups were disinfected with povidone and then dissected. The chest was opened and the heart was rapidly removed and washed with $\mathrm{Ca}^{2+}$ and $\mathrm{Mg}^{2+}$-free phosphate-buffered saline (PBS) solution (pH 7.2, WelGENE). Using microdissecting scissors, the hearts were minced until the pieces were approximately $1 \mathrm{~mm}^{3}$, and then treated with $5 \mathrm{~mL}$ of collagenase type II $(0.9 \mathrm{mg} / \mathrm{mL}, 210$ units $/ \mathrm{mg}$, Gibco BRL) for $7 \mathrm{~min}$ at $20^{\circ} \mathrm{C}$. The cells in the supernatant were transferred to a tube containing cell culture medium $[\alpha-$ minimum essential medium (MEM) containing 10\% fetal bovine serum, WelGENE]. The tubes were centrifuged at $1000 \mathrm{~g}$ for $4 \mathrm{~min}$ at room temperature, and the cell pellet was resuspended in $3 \mathrm{~mL}$ of cell culture medium. The above procedures were repeated $6-8$ times until there was little tissue left. The cell suspensions were washed twice with cell culture medium and seeded to achieve a final concentration of $5 \times 10^{5}$ cells $/ \mathrm{mL}$. Then, they were plated onto gelatin-coated 6-well plates. The cells were cultured in $\alpha$-MEM containing $10 \%$ fetal bovine serum with $0.1 \mathrm{mM}$ Bromodeoxyuridine (BrdU), which was used to prevent proliferation of cardiac fibroblasts. The cells were then cultured in a $5 \% \mathrm{CO}_{2}$ incubator at $37^{\circ} \mathrm{C}$.

Cell viability assay-Neonatal rat cardiomyocytes were plated in triplicate wells of 96-well plates at a density of $4 \times 10^{3}$ cells per well and treated with the designated concentrations of the extracts. Cardiomyocytes have been put in a hypoxic incubator (VISION SCIENTIFIC) maintained at $37^{\circ} \mathrm{C}$ in which ambient oxygen was replaced with a mixture of $5 \% \mathrm{CO}_{2}$ with $1 \%$ $\mathrm{O}_{2}$ balanced with $\mathrm{N}_{2}$ for 30 min prior to exposure to reoxygenation with normoxic condition for 1 hour. Normoxic controls were cultured in a humidified incubator at $37{ }^{\circ} \mathrm{C}$ in the presence of $5 \% \mathrm{CO}_{2}$. Cell viability was assessed using 3-(4,5-dimethylthiazol-2-yl)2,5-diphenyltetrazolium bromide (MTT) assay. After the incubation period, MTT solution (Sigma, St. Louis, MO, USA) was added to each well to a final concentration of $0.5 \mathrm{mg} / \mathrm{mL}$ and incubated at $37^{\circ} \mathrm{C}$ for $3 \mathrm{~h}$ to allow MTT reduction. The formazan crystals were dissolved by adding dimethyl sulfoxide (DMSO), and the absorbance was measured at $570 \mathrm{~nm}$ with a spectrophotometer. The experiments were performed in triplicate.

Immunoblot analysis - Neonatal rat cardiomyocytes were washed in PBS and lysed in RIPA buffer [containing $1 \mathrm{mM}$ phenylmethylsulfonyl fluoride (PMSF) and protease inhibitor cocktail]. Protein concentrations were determined using the Bradford protein assay kit (Bio-Rad, Hercules, CA). Equal quantities of proteins were separated by sodium dodecyl sulfate-polyacrylamide gel electrophoresis (SDSPAGE) in a $10 \%$ gel and transferred to a polyvinylidene difluoride membrane (Bio-Rad laboratories. Inc.). After membrane blocking with Tris-buffered saline-Tween 20 (TBS-T, $0.1 \%$ Tween 20 ) containing $5 \%$ or $10 \%$ skim milk for $1 \mathrm{~h}$ at room temperature, the membrane was incubated $16 \mathrm{~h}$ with primary antibody at $4{ }^{\circ} \mathrm{C}$. The primary antibodies were used at the following dilutions in blocking buffer: SERCA2a (1:4000, Cell Signaling); RyR2 (1:500, Abcam); and phospho CamKII (1:500, Ab frontier). The membrane was washed five times with TBS-T for $5 \mathrm{~min}$ and incubated with secondary antibodies for $1 \mathrm{~h} 30 \mathrm{~min}$ at room temperature. After extensive washing, bands were detected with an enhanced chemiluminescence reagent (ECL, BIONOTE, Animal Genetics Inc.). Band intensities were quantified using the Image $\mathrm{J}$ quantification software.

Measurement of intracellular reactive oxygen species - Intracellular ROS were measured using fluorescent dye technique. Cells were seeded in a 24-well plate with glass cover slips at a density of $5 \times 10^{4}$ cells $/ \mathrm{mL}$ and cultured for $24 \mathrm{~h}$. Then, the cells were treated with EDM 
and subjected to $H / R$ injury. Next, the cells were washed twice with calcium-free PBS and loaded with $\mathrm{H}_{2}$ DCFDA (Invitrogen, USA) and 4',6-diamidino-2-phenylindole (DAPI) diluted with calcium-free warm PBS to a final concentration of $10 \mu \mathrm{M}$ and $50 \mu \mathrm{g} / \mathrm{mL}$, respectively. Subsequently, the cells were incubated for $10 \mathrm{~min}$ at $37^{\circ} \mathrm{C}$ in dark. The probe $\mathrm{H}_{2}$ DCFDA $(10 \mu \mathrm{M})$ entered the cells, and the acetate groups on $\mathrm{H}_{2}$ DCFDA were cleaved by cellular esterases, trapping the non-fluorescent DCF within the cells. Subsequent oxidation by ROS yielded the fluorescent product DCF. Then, the coverslips were gently washed three times in warm PBS and placed in a chamber, which was mounted on the stage of an inverted microscope equipped with a confocal laser-scanning system. The dye, when exposed to an excitation wavelength of $480 \mathrm{~nm}$, emitted light at $535 \mathrm{~nm}$ only when it was oxidized. Fluorescence images were collected using a confocal microscope (Fluoview FV1000 confocal system, Olympus) by excitation at $488 \mathrm{~nm}$ and emission at wavelengths greater than $500 \mathrm{~nm}$ using a long-pass barrier filter. The fluorescence intensity of an equivalent field size $(3 \times 3$ $\mathrm{mm}$ ) in the plate was measured using the Image $\mathrm{J}$ quantification software.

Measurement of intracellular $\mathrm{Ca}^{2+}$ alteration - The intracellular calcium level was measured using the fluorescent calcium indicator Fluo-4am (Invitrogen, USA). Cardiomyocytes were seeded into a 4-well chamber at a density of $1 \times 10^{5}$ cells $/ \mathrm{mL}$ and cultured for $24 \mathrm{~h}$. Then, the cells were treated with EDM and subjected to $H / R$ injury. The cells were washed with serum-free medium $(\alpha$-MEM, WelGENE), loaded with Fluo-4am diluted with serum-free medium to a final concentration of $2 \mu \mathrm{M}$, and incubated for $20 \mathrm{~min}$ at $37^{\circ} \mathrm{C}$ in dark. Subsequently, the cells were washed twice with warm PBS and covered with the cover slide. Fluo-4am fluorescence imaging was performed using confocal microscopy (Fluoview FV1000 confocal system, Olympus). Fluo-4am was excited with a laser at $488 \mathrm{~nm}$, and fluorescence was measured at a wavelength of $515 \mathrm{~nm}$.

Statistical analysis - All quantified data from at least triplicate samples were analyzed with SPSS 13.0 software. Data are expressed as mean \pm SD. Statistical comparisons between two groups were performed using Student's ttest. Statistical comparisons among multiple groups were performed using analysis of variance (ANOVA). A twotailed $\mathrm{P}<0.05$ was considered statistically significant.

\section{Result and Discussion}

To date, several studies demonstrated that hypoxia alone induces time-dependent apoptosis and reoxygenation increases the number of apoptotic cells beyond that in response to hypoxia alone. ${ }^{18}$ In this study, the effects of EDM on H/R-injured cardiomyocytes were investigated. All the samples used in this study are listed in Table 1. Because the EDM did not show any toxicity in cardiomyocytes up to $50 \mu \mathrm{g} / \mathrm{ml}$, the working concentrations were determined with $25 \mu \mathrm{g} / \mathrm{ml}$ in all experiments. As shown in Fig. 1A, cell viability in EDM \#1- and \#2treated groups was respectively 73.4 and $87.7 \%$ in hypoxia (30 $\mathrm{min}) /$ reoxygenation $(1 \mathrm{~h})$-injured cardiomyocytes, demonstrating a significant increase than that in the

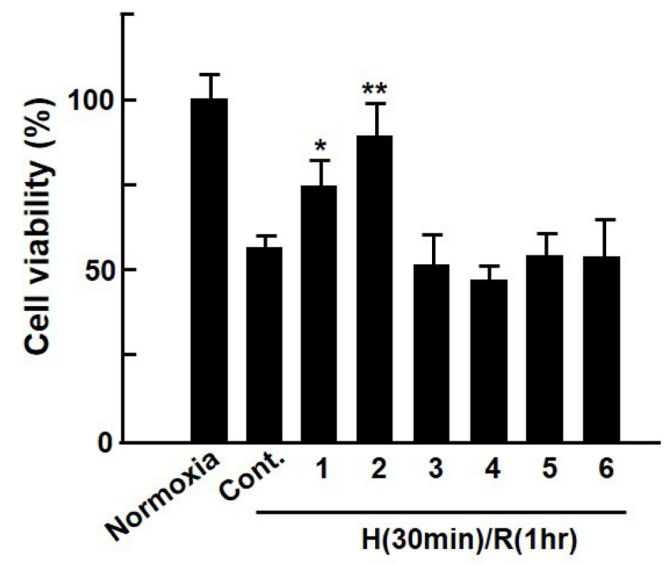

Fig. 1. Protective effect of extract from Dendropanax morbifera on hypoxia/reoxygenation-injured cardiomyocytes. Cardiomyocytes were subjected to hypoxia $(30 \mathrm{~min})$-reoxygenation $(1 \mathrm{~h})$ after $1 \mathrm{~h}$ of Dendropanax morbifera extract (EDM) treatment. The cell viability was evaluated after $24 \mathrm{~h}$ using MTT assay. Values are presented as mean \pm S.D. $* P<0.05$ or ${ }^{* *} P<0.01$ vs. control.

Table 1. Samples prepared and used in this study

\begin{tabular}{|c|c|c|c|}
\hline Extraction process & Sources & Yield (\%) & Sample No. \\
\hline \multirow{2}{*}{ Water ex. (WE) } & Stem & 2.5 & 1 \\
\hline & Leaves & 9.4 & 2 \\
\hline \multirow{2}{*}{ Supercritical fluid ex. (SFE) } & Stem & 3.7 & 3 \\
\hline & Leaves & 7.2 & 4 \\
\hline \multirow{2}{*}{ Extraction after fermentation } & Stem & 2.4 & 5 \\
\hline & Leaves & 8.7 & 6 \\
\hline
\end{tabular}



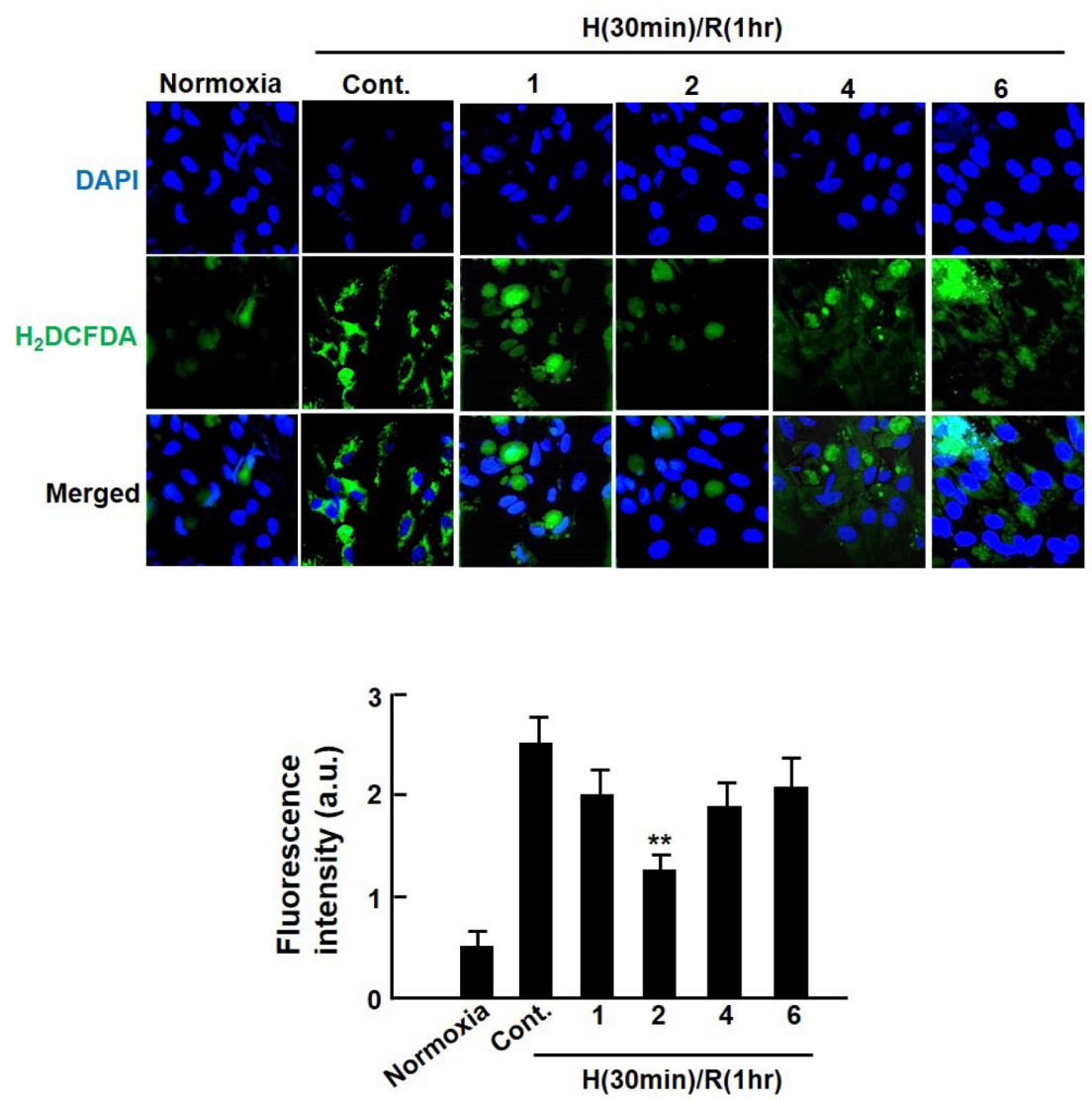

Fig. 2. Inhibitory effect of extract from Dendropanax morbifera on intracellular ROS generation in hypoxia/reoxygenation-injured cardiomyocytes. Cardiomyocytes were subjected to hypoxia $(30 \mathrm{~min})$-reoxygenation $(1 \mathrm{~h})$ after $1 \mathrm{~h}$ of Dendropanax morbifera extract (EDM) treatment. Then, fluorescent $\mathrm{H}_{2}$ DCF-DA images were taken using a confocal laser microscope (Olympus, Japan) at excitation and emission wavelengths of 488 and $520 \mathrm{~nm}$, respectively (fluorescent images were magnified: $\times 400$ ). The fluorescence intensity of an equivalent field size $(2.5 \times 2.5 \mathrm{~mm})$ was measured using the Image $\mathbf{J}$ quantification software. Values are presented as mean \pm S.D. $* * P<0.01$ vs. control.

non-treated group $(56.3 \%)$. However, there were no differences in EDM of \#3-, 4-, 5-, 6-treated groups compared to the non-treated group. EDM \#1 and 2 were prepared using water extraction method as shown in Table 1. These findings suggest that EDM has protective effects on the progress of $\mathrm{H} / \mathrm{R}$ injury-induced apoptosis in cardiomyocytes.

Several studies have demonstrated that the production of ROS plays an important role in H/R-induced cardiomyocyte death. ${ }^{19,20}$ Therefore, to confirm whether EDM has an inhibitory effect on intracellular ROS generation in H/R-injured cardiomyocytes, we evaluated ROS generation in $\mathrm{H} / \mathrm{R}$-injured cardiomyocytes treated with EDM using fluorescence assay method. 2',7'-dichlorofluorescein diacetate (DCF-DA) is a cell-permeable non-fluorescent probe that is de-esterified intracellularly and rapidly oxidized to highly fluorescent DCF in the presence of ROS. There were no differences in EDM of \#3- and 5treated groups compared to the non-treated group (data not shown). Thus, we showed \#1-, 2-, 4-, and 6-treated groups in Fig. 2 and 3. As shown in Fig. 2, treatment of H/R-injured cardiomyocytes with EDM of \#2 dramatically reduced ROS generation compared with positive control, indicating that EDM protects cardiomyocytes against $\mathrm{H} / \mathrm{R}$ injury through the decrease of ROS generation.

Intracellular calcium $\left(\left[\mathrm{Ca}^{2+}\right] \mathrm{i}\right)$ overload induced by chronic hypoxia alters $\left[\mathrm{Ca}^{2+}\right] \mathrm{i}$ homeostasis, which plays an important role in mediating myocardial injury. ${ }^{21} \mathrm{To}$ confirm whether treatment with EDM improves $\mathrm{Ca}^{2+}$ perturbation in $\mathrm{H} / \mathrm{R}$-injured cardiomyocytes, changes in intracellular calcium concentration was measured with Fluo-4am. As shown in Fig. 3, the cytosolic calcium concentration was reduced in $\mathrm{H} / \mathrm{R}$-injured cardiomyocytes treated with EDM \#2 compared with that in non-treated control. This result suggests that EDM ameliorates $\left[\mathrm{Ca}^{2+}\right] \mathrm{i}$ homeostasis, and consequently exerts cardioprotective effects and reduces hypoxic-induced cardiomyocyte damage.

Chronic hypoxia induced defective $\left[\mathrm{Ca}^{2+}\right] \mathrm{i}$ homeostasis 

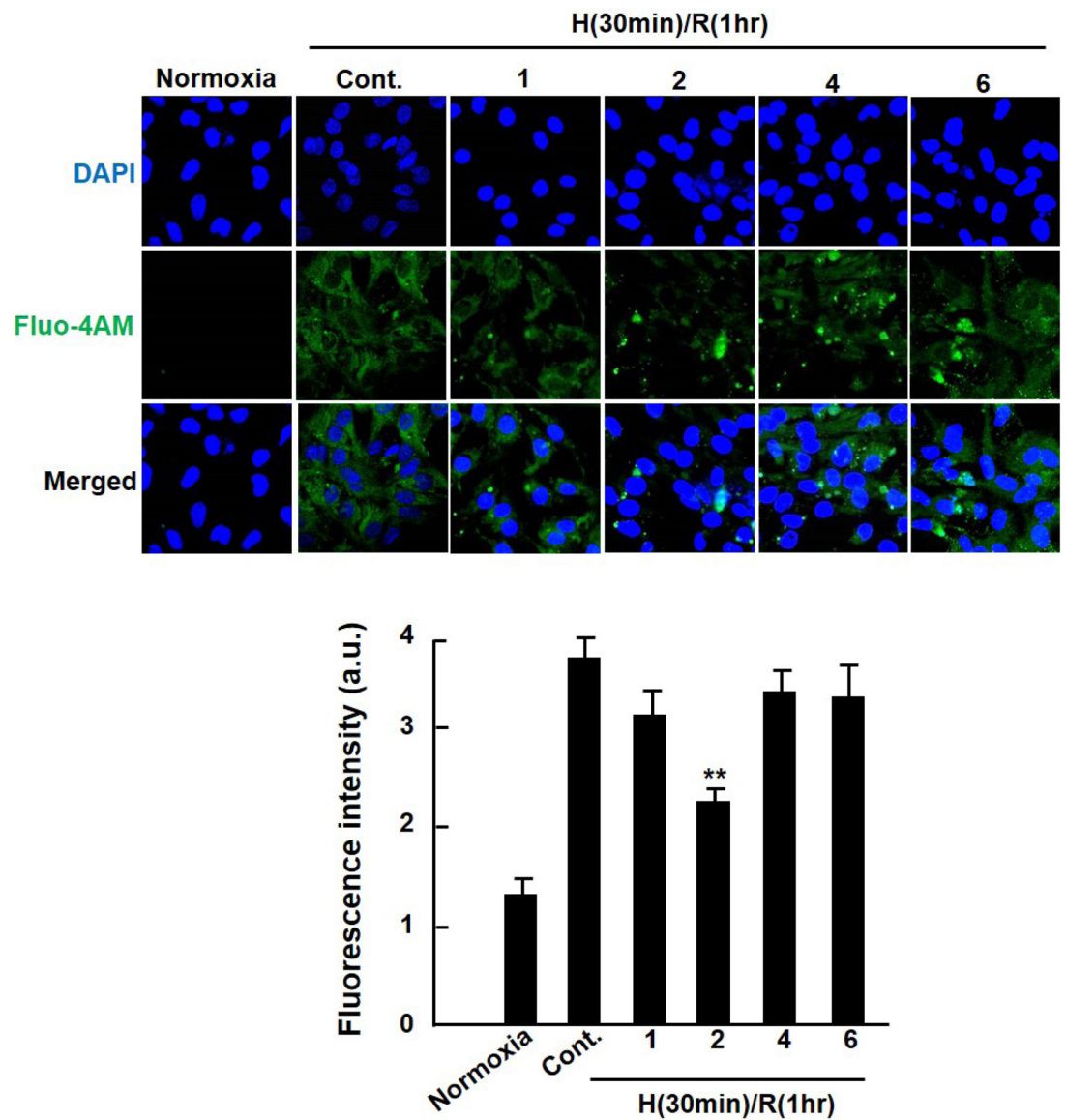

Fig. 3. Effect of extract from Dendropanax morbifera on intracellular calcium perturbation in hypoxia/reoxygenation-injured cardiomyocytes. Cardiomyocytes were subjected to hypoxia $(30 \mathrm{~min})$-reoxygenation $(1 \mathrm{~h})$ after $1 \mathrm{~h}$ of Dendropanax morbifera extract (EDM) treatment. Fluorescent Fluo-4am images were taken by a confocal laser microscope (Olympus, Japan). Values are presented as mean \pm S.D. $* P<0.05$ vs. control.

and increased ROS generation, which play important roles in mediating myocardial injury. ${ }^{22}$ Several ion transport pathways are highly sensitive to redox regulation, and oxidative stress directly impedes intracellular $\mathrm{Ca}^{2+}$ homeostasis. ${ }^{23}$ Downregulation of key $\mathrm{Ca}^{2+}$-handling proteins, such as sarco(endo)plasmic reticulum $\mathrm{Ca}^{2+}$ ATPase (SERCA)2a and ryanodine receptor (RyR2), is a major cause of abnormal $\mathrm{Ca}^{2+}$ homeostasis in cardiomyopathy. $^{24}$ Alterations in SERCA2a and RyR2 expression result in altered cytosolic $\mathrm{Ca}^{2+}$ transients, leading to abnormal contraction. ${ }^{25}$ Recently, it was revealed that oxidative stress can activate calcium calmodulin kinase II (CaMK II). ${ }^{26}$ Therefore, altered expression levels of calcium homeostasis-related proteins by EDM in H/Rinjured cardiomyocytes were evaluated by western blotting. Increased expression of RyR2, phosphorylated CaMKII, and SERCA2a was observed in H/R-injured cardiomyocytes. This increase in expression was significantly restored by \#2 of EDM treatment (Fig. 4). These findings indicate that EDM treatment of $H / R$ injury prevents dysregulation of calcium regulatory proteins in cardiomyocytes.

Acute myocardial infarction induced by ischemiareperfusion is the main cause of death worldwide. The present study demonstrated that EDM ameliorated H/Rinjury induced cardiomyocytes death via inhibition of ROS generation and calcium perturbation. Severe stenosis or occlusion of the coronary artery supplying blood to the heart leads to the blockade of supply of oxygen and nutrients necessary for the survival of myocardial cells, resulting in myocardial cell death or severe myocardial dysfunction. ${ }^{1}$ In case of revascularization of cardiac blood flow by angioplasty, wherein reperfusion of coronary artery with stenosis or occlusion is performed, a sudden increase of ROS in cardiomyocytes and a strong inflammatory reaction led to secondary myocardial cell death. ${ }^{2}$ Subsequently, the calcium concentration changed and calcium-regulating proteins were denatured, inducing 

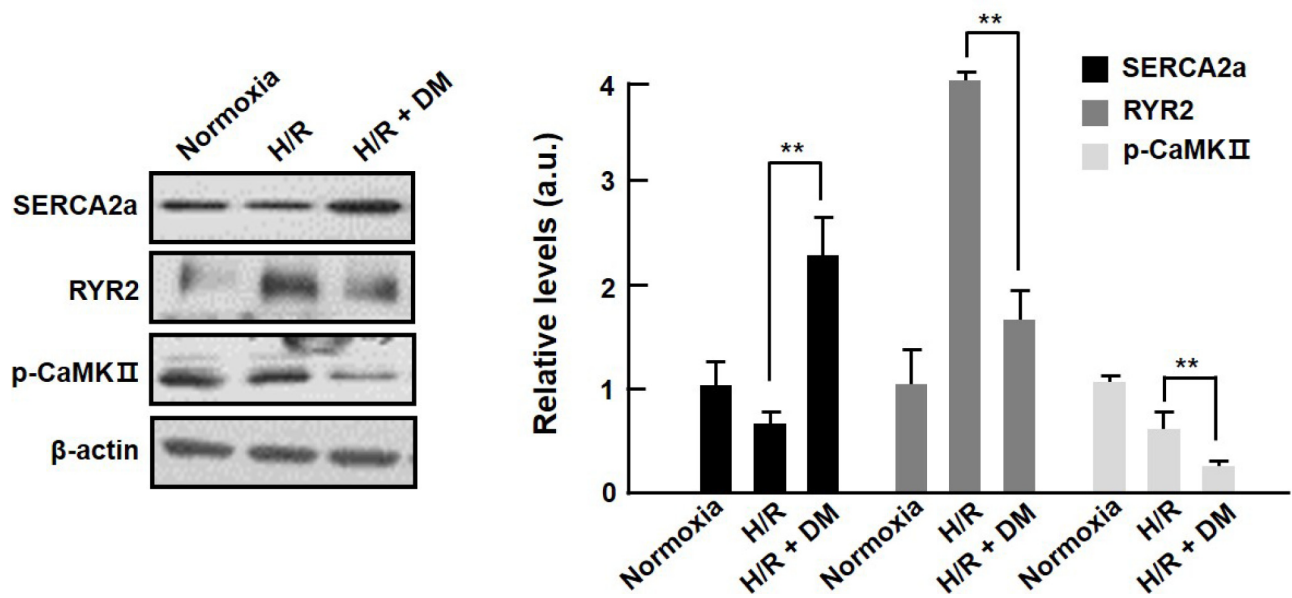

Fig. 4. Altered expression levels of calcium homeostasis-related proteins by extract from Dendropanax morbifera in hypoxia/ reoxygenation-injured cardiomyocytes. Cardiomyocytes were subjected to hypoxia $(30 \mathrm{~min})$-reoxygenation $(1 \mathrm{~h})$ after $1 \mathrm{~h}$ treatment with \#2 of Dendropanax morbifera extract (EDM). The levels of SERCA2a, p-CaMKII, and RyR2 were evaluated by western blotting. Values are presented as mean \pm S.D. ${ }^{* *} P<0.01 v$ s. control.

abnormal function in myocardial cells. Therefore, it is important to maintain normal myocardial function by inhibiting the ROS generation and calcium perturbation causing myocardial cell death. Materials derived from natural products have recently been actively developed for the treatment of CVD. Owing to their safety, low toxicity, and general acceptance as dietary supplements, natural dietary elements have been investigated in detail for the prevention of several diseases, such as cancer, diabetes, and CVD. ${ }^{27}$ The extracts from the stem of $D$. morbifera facilitated cadmium excretion from the blood and the kidneys of cadmium-exposed rats and inhibited cadmium-induced oxidative stress in the brain through increased levels of antioxidants. ${ }^{28}$ The ethanol extracts of D. morbifera markedly inhibited the growth of human leukemia-U937 cells through the reduction of cell proliferation and induction of apoptosis through caspasedependent pathway. ${ }^{29}$ It has been reported that essential oils from $D$. morbifera have significant lipid-lowering effects. ${ }^{9}$ However, the effect of EDM on CVD is unknown.

In the present study, we demonstrated that EDM inhibits $\mathrm{H} / \mathrm{R}$ injury-induced cardiomyocyte death through inhibition of ROS generation and $\mathrm{Ca}^{2+}$ perturbation. The results showed that the altered expression levels of calcium homeostasis-related proteins, namely SERCA2a, RyR2, and p-CamKII, were successfully rescued by $\mathrm{EDM}$, demonstrating its effect in suppressing the increase in calcium ion concentration in damaged cardiomyocytes. In addition, the cardioprotective effects of EDM differed depending on the extraction method and resources. EDM obtained from leaves by water extraction method has been shown the highest cardioprotective effect in this study. Our results provided conclusive evidence that EDM successfully inhibited $\mathrm{H} / \mathrm{R}$ injury-induced cardiomyocyte death. However, further investigations on the chemical component responsible for these effects, the mechanisms underlying the regulation of calcium ion channel, and the signaling pathways that participate in the process of cardioprotective effects are warranted to allow the future use of EDM in the treatment of CVD. In addition, this study was conducted only in vitro, so further investigations will be needed to evaluate the effect of EDM on ischemia/ reperfusion-injured myocardium in in vivo.

\section{Acknowledgments}

This work was supported by the grants from the Chosun University, 2017.

\section{Referances}

(1) Hausenloy, D. J.; Yellon, D. M. J. Clin. Invest. 2013, 123, 92-100.

(2) Cadenas, S.; Aragonés, J.; Landázuri, M. O. Cardiovasc. Res. 2010, 88, 219-228.

(3) Sabharwal, S. S.; Schumacker, P. T. Nat. Rev. Cancer 2014, 14, 709721.

(4) Sugamura, K.; Keaney, J. F. Jr. Free Radic. Biol. Med. 2011, 51, 978-992.

(5) Viña, J.; Borras, C.; Abdelaziz, K. M.; Garcia-Valles, R.; GomezCabrera, M. C. Antioxid. Redox Signal. 2013, 19, 779-787.

(6) Duchen, M. R.; Verkhratsky, A.; Muallem, S. Cell Calcium 2008, 44, 1-5.

(7) Yu, H. Y.; Kim, K. S.; Lee, Y. C.; Moon, H. I.; Lee, J. H. Evid. Based Complement. Alternat. Med. 2012, 2012, 637512.

(8) Park, B. Y.; Min, B. S.; Oh, S. R.; Kim, J. H.; Kim, T. J.; Kim, D. 
H.; Bae, K. H.; Lee, H. K. J. Ethnopharmacol. 2004, 90, 403-408.

(9) Moon, H. I. Hum. Exp. Toxicol. 2011, 30, 870-875.

(10) Chung, I. M.; Kim, M. Y.; Park, W. H.; Moon, H. I. Pharmazie 2009, 64, 547-549.

(11) Chung, I. M.; Kim, M. Y.; Park, S. D.; Park, W. H.; Moon, H. I. Phytother. Res. 2009, 23, 1634-1637.

(12) Chung, I. M.; Song, H. K.; Kim, S. J.; Moon, H. I. Phytother. Res. 2011, 25, 784-786.

(13) Akram, M.; Kim, K. S.; Kim, E. S.; Syed, A. S.; Kim, C. Y.; Lee, J. S.; Bae, O. N. Biol. Pharm. Bull. 2016, 39, 728-736.

(14) Yu, H. Y.; Jin, C. Y.; Kim, K. S.; Lee, Y. C.; Park, S. H.; Kim, G. Y.; Kim, W. J.; Moon, H. I.; Choi, Y. H.; Lee, J. H. J. Agric. Food Chem. 2012, 60, 5400-5406.

(15) Lee, J. W.; Kim, K. S.; An, H. K.; Kim, C. H.; Moon, H. I.; Lee, Y. C. PLoS One. 2013, 8, e83611.

(16) Jin, C. Y.; Yu, H. Y.; Park, C.; Han, M. H.; Hong, S. H.; Kim, K. S.; Lee, Y. C.; Chang, Y. C.; Cheong, J.; Moon, S. K.; Kim, G. Y.; Moon, H. I.; Kim, W. J.; Lee, J. H.; Choi, Y. H. Int. J. Oncol. 2013, 43, 19431950 .

(17) Vandergriff, A. C.; Hensley, M. T.; Cheng, K. J. Vis. Exp. 2015, 98, e52726.

(18) Buja, L. M.; Entman, M. L. Circulation 1998, 98, 1355-1357.

(19) Griendling, K. K.; FitzGerald, G. A. Circulation 2003, 108, 20342040.

(20) Schriewer, J. M.; Peek, C. B.; Bass, J.; Schumacker, P. T. J. Am. Heart Assoc. 2013, 2, e000159.

(21) Barry, W. H.; Bridge, J. H. Circulation 1993, 87, 1806-1815.
(22) Choi, K. M.; Zhong, Y.; Hoit, B. D.; Grupp, I. L.; Hahn, H.; Dilly, K. W.; Guatimosim, S.; Lederer, W. J.; Matlib, M. A. Am. J. Physiol. Heart Circ. Physiol. 2002, 283, H1398- H1408.

(23) Boudina, S.; Abel, E. D. Circulation. 2007, 115, 3213-3223.

(24) Trost, S. U.; Belke, D. D.; Bluhm, W. F.; Meyer, M.; Swanson, E.; Dillmann, W. H. Diabetes 2002, 51, 1166-1171.

(25) Kain, V.; Kumar, S.; Sitasawad, S. L. Cardiovasc. Diabetol. 2011, 10,97 .

(26) Erickson, J. R.; Joiner, M. L.; Guan, X.; Kutschke, W.; Yang, J.; Oddis, C. V.; Bartlett, R. K.; Lowe, J. S.; O'Donnell, S. E.; Aykin-Burns, N.; Zimmerman, M. C.; Zimmerman, K.; Ham, A. J.; Weiss, R. M.; Spitz, D. R.; Shea, M. A.; Colbran, R. J.; Mohler, P. J.; Anderson, M. E. Cell 2008, 133, 462-474.

(27) Li, Y. H.; Chung, H. C.; Liu, S. L.; Chao, T. H.; Chen, J. C. Int. Heart J. 2009, 50, 207-220.

(28) Kim, W.; Kim, D. W.; Yoo, D. Y.; Jung, H. Y.; Nam, S. M.; Kim, J. W.; Hong, S. M.; Kim, D. W.; Choi, J. H.; Moon, S. M.; Yoon, Y. S.; Hwang, I. K. BMC. Complement. Altern. Med. 2014, 14, 428.

(29) Lee, J. W.; Park, C.; Han, M. H.; Hong, S. H.; Lee, T. K.; Lee, S. H.; Kim, G. Y.; Choi, Y. H. Oncol. Rep. 2013, 30, 1231-1238.

Received September 21, 2018 Revised December 12, 2018 Accepted December 12, 2018 